\title{
The spectrum of cerebral vasoconstriction: a diagnostic conundrum
}

Volume 4 Issue I - 2016

\section{Editorial}

Although rare, the co-occurrence of Reversible Cerebral Vasoconstriction Syndrome (RCVS) has occurred with subarachnoid hemorrhage (SAH) and Reversible Posterior Leukoencepahlopathy Syndrome (RPLS) or Posterior Reversible Encephalopathy Syndrome (PRES). ${ }^{1}$ Consequently, clinicians are faced with the dilemma of treating concurrent RCVS with RPLS and SAH. In a prospective series of 67 patients with RCVS, cortical SAH, intracerebral hemorrhages (ICH), and RPLS were observed as acute complications of RCVS, whereas cerebral ischemia was a delayed complication. ${ }^{2}$ This sequence of complications leads to a crucial question: how to manage RCVS with active cerebral vasospasm $(\mathrm{CV})$, with con-current SAH and/or RPLS as its early complication, followed by delayed ischemic strokes.

It is well recognized that treatment of hypertension is critical in the early management of ICH, aneurysmal SAH, and RPLS. This creates a dilemma in that aggressive hypertension management can place a patient at risk of ischemia due to cerebral hypoperfusion. There is no specific blood pressure control and CV management guidelines in RCVS, which creates the challenge of treating patients with RCVS who also suffer from delayed ischemic infarcts from CV. It is important to note that CV in RCVS is expected to last up to 12 weeks, ${ }^{1}$ whereas $\mathrm{CV}$ in aneurysmal SAH is known to last up to four weeks. ${ }^{3}$ In aneurysmal SAH, there is a clear guideline that emphasizes the importance of calcium channel blocker (CCB) treatment with the "Triple-H" therapy of hypertension, hypervolemia, and hemodilution to treat active vasospasms and prevent delayed cerebral ischemia. ${ }^{4}$

In aneurysmal SAH, blood in the site of the rupture is known to cause CV close to the aneurysm rupture. ${ }^{5}$ In SAH due to RCVS, the hemorrhage is known to be cortical in location, with extensive and multifocal vasoconstriction of medium- to large-sized arteries away from the site of the hemorrhage. Also, dysregulation in the cerebrovascular autoregulation and rupture of small arterioles due to acute hypertension are thought to cause cortical SAH in RCVS. ${ }^{6}$ Interestingly, RPLS is also thought to affect small-sized arterioles, ${ }^{7}$ leading to the observation of co-occurrence of the RCVS and RPLS in these instances. ${ }^{8}$

The relationship between RCVS and RPLS is thought to be a spectrum of similar diseases that share the similar pathophysiology of dysregulation in cerebral arterial tone. ${ }^{9}$ RPLS is also known to be an inaccurate terminology as it can also present with diffuse vasoconstriction..$^{10}$ Although management of hypertension in RPLS reportedly leads to resolution of symptoms, rapid correction of hypertension can potentially cause cerebral ischemia. ${ }^{11}$

It is also important to note that there is no specific management guideline that supports the specific duration of CCB in RCVS or the superiority of one CCB to another. Since the duration of RCVS can be prolonged, one can consider continuing a $\mathrm{CCB}$ for one to three months - although this does not change the time course of vasospasms, ${ }^{12}$ or

\author{
Adalia H Jun-O'Connell, Banu Sundar, Majaz \\ Moonis \\ Department of Neurology, UMass Memorial Medical Center/ \\ UMass Medical School, USA
}

Correspondence: Majaz Moonis MD, DM, MRCP (i), FRCP (Edinburgh), FAAN, FAHA, FAASM, Professor of Neurology, Director of UMassmemorial stroke services, UMass Memorial Medical Center/UMass Medical School, 55 Lake Ave North, Worcester MA 01655, USA,

Email majaz.moonis@umassmemorial.org

Received: January 10, 2016 | Published: January 12, 2016

until either the follow-up four-vessel angiography shows evidence of resolution or a complete symptom resolution is achieved. ${ }^{2}$

The effectiveness of nimodipine in RCVS is reportedly between $64 \%,{ }^{2}$ and $83 \% .{ }^{13}$ Intravenous (IV) nimodipine $(0.5-2 \mathrm{mg} / \mathrm{hr})$ has been recommended with close blood pressure monitoring in an intensive care unit in acute phase. However, there is no literature data to support superiority of IV CCB over oral CCB, and hypotension needs to be avoided, given the risk of ischemic complications. It has been suggested to correct hypotension, especially if SBP is less than $100 \mathrm{~mm} \mathrm{Hg},{ }^{14}$ although there is no evidence-based data to support this.

Finally, intra-arterial (IA) vasodilators and balloon angioplasty can be safely used in refractory cases. ${ }^{15}$ Recurrent IA nimodipine administration with angioplasty has been described to be safe and effective in refractory RCVS. ${ }^{16}$ IA verapamil and milrinone (a phosphodiesterase inhibitor) also have been shown to be helpful in refractory cases.$^{17}$ Certainly, a clinical trial may confirm if this can be safely replicated in such complex and challenging cases. IA CCB are known to be safe and potent in treating refractory cases and can be superior to oral or IV CCB treatment due to rapid effect on cerebral arterial vasodilation and reduced risk of systemic hypotension. ${ }^{18,19}$

Clinicians need to be aware of various manifestations of cerebral arterial vasoconstriction which does not always imply RCVS but may represent a spectrum ranging from migraine, RPLS, RCVS and potentially more serious conditions like isolated or systemic cerebral angiitis. "Reversible" in RCVS is misleading as it can still lead to devastating complications, especially ICH and ischemic strokes, despite the maximal medical management. Therefore, there is a need for close monitoring during all phases of RCVS, as if one would attend closely to aneurysmal SAH. 


\section{Acknowledgments}

None.

\section{Conflicts of interest}

None.

\section{References}

1. Dodick DW, Eross EJ, Drazkowski JF, et al. Thunderclap headache associated with reversible vasospasm and posterior leukoencephalopathy syndrome. Cephalagia. 2003;23(10):994-999.

2. Ducros A, Boukobza M, Porcher R, et al. The clinical and radiological spectrum of reversible cerebral vasoconstriction syndrome. A prospective series of 67 patients. Brain. 2007;130(Pt 12):3091-3101.

3. Lee KH, Lukovits T, Friedman JA. "Triple-H" therapy for cerebra vasospasm following subarchnoid hemorrhage. Neurocrit Care. 2006;4(1):68-76.

4. Connolly ES, Rabinstein AA, Carhuapoma JR, et al. Guidelines for the management of aneurysmal subarachnoid hemorrhage: a guideline for healthcare professionals from the American Heart Association/american Stroke Association. Stroke. 2012;43(6):1711-1737.

5. Noda K, Fukae J, Fujishima K, et al. Reversible cerebral vasoconstriction syndrome presenting as subarachnoid hemorrhage, reversible posterior leukoencephalopathy, and cerebral infarction. Intern Med. 2011;50(11):1227-1233.

6. Shah AK. Non-aneurysmal primary subarachnoid hemorrhage in pregnancy-induced hypertension and eclampsia. Neurology. 2003;61(1):117-120.

7. Singhal AB. Postpartum angiopathy with reversible posterior leukoencephalopathy. Arch Neurol. 2004;61(3):411-416.

8. Chen SP, Fuh JK, Wang SJ. Reversible cerebral vasoconstriction syndrome: current and future perspectives. Expert Rev Neurother. 2011;11(9):1265-1276.
9. Agarwal R, Davis C, Altinok D, et al. Posterior reversible encephalopathy and cerebral vasoconstriction in a patient with hemolytic uremic syndrome. Pediatr Neurol, 2014;50(5):518-521.

10. Bartynski WS, Boardman JF. Catheter angiography, MR angiography and MR perfusion in posterior reversible encephalopathy syndrome. AJNR Am J Neuroradiol. 2008;29(3):447-455.

11. Fugate JE, Rabinstein AA. Posterior reversible encephalopathy syndrome: clinical and radiological manifestions, pathophysiology, and outstanding questions. Lancet Neurol. 2015;4(9):914-925.

12. Ducros A. Reversible cerebral vasoconstriction syndrome. Lancet Neurol. 2012;11(10):906-917.

13. Chen SP, Fuh JL, Lirng JF, et al. Recurrent primary thunderclap headache and benign CNS angiopathy: spectra of the same disorder? Neurology. 2006;67(12):2164-2169.

14. Chen SP, Fuh JL, Wang SJ. Reversible cerebral vasoconstriction syndrome: an under-recognized clinical emergency. Ther Adv Neurol Disord. 2010;3(3):161-171.

15. Velez A, McKinney JS. Reversible cerebral vasoconstriction syndrome: a review of recent research. Curr Neurol Neurosci Rep. 2013;13(1):319.

16. Ioannidis I, Nasis N, Agianniotaki E, et al. Reversible cerebral vasoconstriction syndrome: treatment with multiple session of intra-arterial nimodipine and angioplasty. Interv Neuroradiol. 2012;18(3):297-302.

17. Farid H, Tatum JK, Wong C, et al. Reversible cerebral vasoconstriction syndrome: treatment with combined intra-arterial verapamil infusion and intracranial angioplasty. AJNR Am J Neuroradiol. 2011;32(10):E184 E187.

18. Bouchard M, Verreault S, Gariépy JL, et al. Intra-arterial milrinone for reversible cerebral vasoconstriction syndrome. Headache. 2009;49(1):142-145.

19. Farid H, Tatum JK, Wong C, et al. Reversible cerebral vasoconstriction syndrome: treatment with combined intra-arterial verapamil infusion and intracranial angioplasty. Am J Neuroradiol. 2011;32(10):E184 E187. 